\title{
Long-term effects of breast-feeding in a national birth cohort: educational attainment and midlife cognitive function
}

\author{
Marcus Richards*, Rebecca Hardy and Michael EJ Wadsworth \\ MRC National Survey of Health and Development, Royal Free \& University College Medical School, University \\ College London, Department of Epidemiology and Public Health, 1-19 Torrington Place, London WC1E 6BT, UK
}

Submitted 5 December 2001: Accepted 5 March 2002

\begin{abstract}
Objective: A recent meta-analysis showed that breast-feeding confers a 3.2 point increment in cognitive function through adolescence. Little is known, however, about possible longer-term effects of breast-feeding. We investigated the effect of breastfeeding on educational attainment, and on a range of cognitive skills in midlife, in the British 1946 birth cohort.

Design: Regression analyses were used to test the association between breast-feeding, likelihood of obtaining advanced educational qualifications by age 26 years, and three cognitive test scores at age 53 years: i.e. reading ability (NART), timed visual search and verbal memory. These associations were then adjusted for social confounding variables and for cognitive ability at age 15 years.

Setting and subjects: One thousand seven hundred and thirty-nine male and female participants in the MRC National Survey of Health and Development, also known as the British 1946 birth cohort, distributed throughout England, Wales and Scotland. Results: Breast-feeding was significantly and positively associated with educational attainment, an effect that was independent of early social background, but largely accounted for by cognitive ability at age 15 years. Breast-feeding was significantly and positively associated with the NART at 53 years, an effect that was independent of early social background, educational attainment and adult social class, but, again, largely accounted for by cognitive ability at 15 years. There was no independent effect of breast-feeding on timed visual search or verbal memory at 53 years.

Conclusion: The benefit of breast-feeding has long-term potential impact across the life course through its influence on childhood cognition and educational attainment.
\end{abstract}

\section{Keywords \\ Breast-feeding \\ Educational attainment \\ Cognitive function \\ Birth cohort}

There is a broad consensus that breast-feeding modestly benefits cognitive development, independent of social background. A recent meta-analysis showed a benefit of 3.2 points in cognitive function after adjusting for relevant covariates ${ }^{1}$. However, this meta-analysis only included studies that tested cognitive function between infancy and adolescence, and little is known about possible longer-term effects of breast-feeding. The MRC National Survey of Health and Development, also known as the British 1946 birth cohort, allows such an investigation. First, we investigated whether breastfeeding was associated with educational attainment. Second, we investigated long-term effects of breastfeeding on cognitive function itself, and whether any association was mediated by the effects of breastfeeding on education. Using data from this cohort, Rodgers originally showed a beneficial effect of breastfeeding on a test of verbal comprehension at age 15 years, after adjusting for a variety of family background variables $^{2}$. Now, in the same study, cognitive function has been measured on several occasions in adulthood, most recently at age 53 years, when a range of cognitive skills was assessed, including those of verbal ability, verbal memory, and mental speed and concentration. We investigated the association between breast-feeding and these skills, for two reasons. First, verbal memory and mental speed and concentration had not been assessed in childhood or early adulthood, and had not been studied in this cohort in relation to breast-feeding. Second, we wished to investigate whether the benefit of breast-feeding on verbal ability found in adolescence was still detectable in midlife. In this regard, we assessed three possibilities: (1) that the benefit was not detectable (perhaps because those who had not been breast-fed caught up during adulthood, or because the effects of breast-feeding had become overshadowed by adult influences); (2) that the benefit was detectable at 53 years but mediated by adolescent ability; and (3) that the benefit detected at 53 years was greater than could be explained by adolescent ability (perhaps because of additive or cumulative benefit from education, occupation or other adult factors). 


\section{Methods}

\section{Participants}

Participants were drawn from the MRC National Survey of Health and Development (NSHD), also known as the British 1946 birth cohort. The survey initially consisted of 5362 children of non-manual and agricultural workers and a random sample of one in four of manual workers selected from all births that occurred in England, Scotland and Wales during one week in March $1946^{3}$. Information about sociodemographic factors and medical, cognitive and psychological function has been repeatedly obtained by interview and examination, most recently in 1999 at age 53 years when sample size was 3035. At age 43 the sample interviewed was shown to be representative, in most respects, of the UK population legitimately and singly born in the immediate post-war era ${ }^{4}$. Exceptions were an over-representation among non-responders of the never married, the least literate, those always in manual social class circumstances, and those with psychiatric illness.

All three of the cognitive tests at age 53 (described below) were completed by 2371 cohort members. Those with no cognitive test scores at this age had significantly poorer cognitive ability at age 15 years $(P<0.001)$ than those with at least one score. Sample size was reduced to 1739 when selected for non-missing values on the potential confounding variables (see below) and cognitive ability at age 15 . Those with missing data for any of these variables had significantly poorer cognitive ability at age 8 years $(P=0.003)$ than those entered into the analyses.

\section{Breast-feeding}

Information about infant feeding was obtained by interview from mothers when the cohort members were aged 2 years. Using this information, duration of breastfeeding was classified as never ( $n=390)$, up to 2 months $(n=369), 2-7$ months $(n=470)$ or $>7$ months $(n=$ 510 ), which allowed approximately equal numbers of participants in each category.

\section{Educational attainment}

The highest educational or training qualification achieved by 26 years of age was classified by the Burnham scale and grouped into ordinary secondary qualifications (' $O$ ' levels and their training equivalents) or less, vs. advanced secondary education ('A' levels and their equivalents) or higher education (degree level or equivalent).

\section{Cognitive measures}

At 53 years cognitive function was assessed by measures of reading ability, verbal memory, verbal fluency, attention, speed and concentration. From this battery three cognitive functions were selected as outcomes for the present study: reading ability, measured by the National Adult Reading Test $\left(\right.$ NART) ${ }^{6}$; verbal memory, measured by a word-list learning task; and mental speed and concentration, measured by a timed letter search task. The NART assesses ability to pronounce 50 words of increasing difficulty, is strongly predictive of full-scale $\mathrm{IQ}^{6,7}$ and is relatively stable across the life course ${ }^{8}$. Verbal memory was chosen because it predicts clinically significant cognitive decline in ageing ${ }^{9,10}$. More generally, many if not most aspects of daily living depend on healthy memory function. This was assessed by a 15 -item word learning task devised by the NSHD. Each word was shown for two seconds. When all 15 words were shown, the respondent was asked to write down as many of the words as possible from memory. This was repeated twice more. Total number of words correctly recalled over the three trials was summed to provide an overall score $($ maximum $=45$ ). Timed visual search, the third cognitive outcome, provided a contrasting outcome, and was assessed by a task requiring participants to cross out the letters $\mathrm{P}$ and $\mathrm{W}$, randomly embedded within a page of other letters, as quickly as possible within one minute. Score was the total number of letters searched (maximum $=600$ ), minus the number of targets missed. All tests at 53 years were administered by a research nurse, following standardised training.

Cognitive ability at age 15 years was used as a controlling variable. This was represented by the sum of three test scores ${ }^{11}$ : Group Ability Test AH4 (verbal and non-verbal intelligence), Watts-Vernon Reading Test (verbal comprehension), and mathematics (arithmetic, geometry, trigonometry and algebra).

\section{Potential confounding variables}

Potential confounders in childhood were sex and measures of family background (assessed by measures of paternal social class, maternal education, birth order, parental interest in education, and material home conditions). Paternal social class was assigned using the fathers' occupation (classified as professional, managerial, intermediate, skilled manual, semi-skilled manual, and unskilled) when the participant was aged 11 years, or, if this was unknown, occupation at age 4 years or 15 years ${ }^{12}$. Maternal education was classified into those with primary or secondary education only with no formal qualifications and those with formal qualifications or any further education. Parental interest in education of the cohort members was represented on a scale of $0-50$, based partly on teacher comments at the end of the first and at the end of the fourth primary school year, and partly on records of the number of times each parent visited the school to discuss their child's progress with the head or class teacher $^{13}$. Material home conditions were represented on a scale of $0-8$, based on ratings, made by a health visitor when cohort members were aged 4 years, of shoes, clothing and cleanliness of the child, age, state of repair, cleanliness and crowdedness of the dwelling, and managing ability of the mother ${ }^{2}$.

Potential adult confounders for the association between breast-feeding and cognition at 53 years were socio- 
Table 1 Early background variables and adult social class by duration of breast-feeding

\begin{tabular}{lccccc}
\hline Variable & Never & Up to 2 months & $2-7$ months & $>7$ months & $P$-value \\
\hline Sex (\% female) & 51.0 & 47.2 & 51.5 & 53.5 & 0.32 \\
Father's social class (\% manual) & 60.5 & 65.9 & 56.8 & 56.3 & 0.02 \\
Mother's education (\% no qualification) & 70.3 & 64.0 & 60.9 & 55.5 & $<0.001$ \\
Adult social class (\% manual) & 36.2 & 34.1 & 30.6 & 30.0 & 0.17 \\
Birth order (mean \& SD) & $2.1(1.3)$ & $1.8(1.1)$ & $2.1(1.2)$ & $2.1(1.2)$ & 0.005 \\
Parental interest in education (mean \& SD) & $30.9(13.6)$ & $30.7(13.4)$ & $32.7(13.8)$ & $33.7(13.5)$ & 0.002 \\
Material home conditions (mean \& SD) & $5.6(1.9)$ & $5.4(1.9)$ & $5.6(1.9)$ & $5.8(1.8)$ & 0.02 \\
\hline
\end{tabular}

SD - standard deviation.

economic status (assessed by social class at 53 years, or earlier if this was unavailable, using similar categories to those for paternal occupational social class) and own educational attainment, coded as above.

\section{Statistical methods}

The association between breast-feeding and educational attainment was investigated using logistic regression. This was done in three stages. First, the unadjusted association between breast-feeding and education was obtained. Second, this was adjusted for the early background variables (see above). Third, the association was further adjusted for cognitive ability at age 15 years. Due to a nonlinear relationship between duration of breast-feeding and educational attainment, breast-feeding duration was entered as a categorical variable (0, up to 2 months, 2-7 months and $>7$ months)

The association between breast-feeding and cognitive function at age 53 years was investigated using separate linear regression models for each cognitive score. This was done using breast-feeding duration as a categorical variable ( 0 , up to 2 months, $2-7$ months and $>7$ months), adjusting for confounders in similar stages to that of the analysis of educational attainment above. In addition, further adjustment for educational attainment and adult social class was performed.

\section{Results}

\section{Breast-feeding and background variables}

Table 1 shows the background variables by duration of breast-feeding. There were no differences at the 5\% level in duration of feeding according to own sex or own social class. However, longer duration was associated with nonmanual social class of origin, positive maternal educational attainment, and increased parental interest in education. Associations between duration of feeding and birth order and material home conditions were significant at the $5 \%$ level, although no clear gradient was observed in either case.

\section{Educational attainment}

Table 2 shows odds ratios (ORs) for obtaining advanced educational qualifications by age 26 years for each category of breast-feeding duration.

The unadjusted association was statistically significant, and the association was J-shaped, with odds of obtaining higher qualifications lowest in those breast-fed for up to 7 months but highest in those breast-fed for longer. The effect of breast-feeding on education was strengthened after adjusting for the early background variables, and a dose-response relationship was observed, the association remaining significant at the 5\% level. For example, for second-born men with maximum disadvantage on all of the social background variables, the predicted percentage obtaining higher educational qualifications was $9.6 \%$ for those who were never breast-fed, compared with $14.3 \%$ for those breast-fed for 7 months or longer. The association was no longer significant, however, after further adjustment for cognitive ability at age 15 years, due to the reduction of the OR for breast-feeding for 7 months or more compared with no breast-feeding.

Table 2 Odds ratios (95\% confidence intervals) and overall $P$-values for likelihood of obtaining advanced educational qualifications for each level of breast-feeding duration. Unadjusted effects are shown, then progressively adjusted for early background ${ }^{\star}$ and cognitive ability at age 15 years

\begin{tabular}{|c|c|c|c|c|c|c|}
\hline \multirow[b]{2}{*}{ Breast-feeding duration } & \multicolumn{2}{|c|}{ Unadjusted } & \multicolumn{2}{|c|}{ +Early background* } & \multicolumn{2}{|c|}{+ Cognitive ability at age 15} \\
\hline & OR $(95 \% \mathrm{Cl})$ & $P$-value & OR $(95 \% \mathrm{Cl})$ & $P$-value & OR $(95 \% \mathrm{Cl})$ & $P$-value \\
\hline & & $<0.001$ & & 0.03 & & 0.18 \\
\hline Never (reference) $(n=390)$ & 1.0 & & 1.0 & & 1.0 & \\
\hline Up to 2 months $(n=369)$ & $0.72(0.54,0.96)$ & & $1.11(0.78,1.58)$ & & $1.02(0.70,1.51)$ & \\
\hline $2-7$ months $(n=470)$ & $0.79(0.59,1.05)$ & & $1.31(0.94,1.82)$ & & $1.25(0.87,1.79)$ & \\
\hline$>7$ months $(n=510)$ & $1.24(0.96,1.60)$ & & $1.58(1.15,2.18)$ & & $1.40(0.98,1.99)$ & \\
\hline
\end{tabular}

* Sex, father's social class, mother's education, birth order, parental interest in education and material home conditions. 


\section{Cognitive function}

Table 3 shows regression coefficients $(R)$, along with 95\% confidence intervals (95\% CI) and $P$-values, for the effect of each breast-feeding duration category ( 0 , up to 2 months, 2-7 months or $>7$ months) on cognitive scores at age 53 years.

The coefficients for the unadjusted effect show that breast-feeding was significantly associated with the NART and with verbal memory, with the former showing the stronger effect. A dose-response effect was evident in both cases. Breast-feeding was not associated with visual search speed.

Coefficients were clearly reduced after adjusting for the early background variables (sex, father's social class, mother's education, birth order, parental interest in education and material home conditions). They were further reduced after adjusting for educational attainment and adult social class, with only that for the NART remaining significant at the 5\% level, but with the unadjusted dose-response effect clearly attenuated. Coefficients for all three tests were further reduced after final adjustment for cognitive ability at age 15 years, and the effect of breast-feeding on the NART was no longer significant at the 5\% level. At this final stage of the model, the difference in NART score, given cognitive ability at age 15 years, between those never breast-fed and those breastfed for more than 7 months was only 0.97 , i.e. less than one word. Thus there was little evidence of any benefit of breast-feeding on reading ability in midlife beyond its effects on cognition during childhood.

Breast-feeding was associated with three out of four of the individual component scores of the cognitive ability score at age 15 years (verbal comprehension, mathematics and AH4 non-verbal ability) at the 5\% level, after controlling for the early background variables. The exception was AH4 verbal ability, which was of borderline significance $(P=0.06)$.

\section{Discussion}

In a longitudinal population-based study we found that duration of breast-feeding was significantly and positively associated with likelihood of obtaining advanced educational qualifications, after controlling for early social background. This effect was explained by that of breastfeeding on adolescent cognition, although it was independent of parental interest in education, an important potential confounder. Breast-feeding was also associated with adult reading ability, as measured by the NART at age 53 years, an effect that was also independent of early social background, as well as independent of educational attainment and adult occupational social class. There was no evidence, however, that breast-feeding was associated with increased reading ability across adulthood, i.e. conferred any benefit beyond its effects on cognition during adolescence. Nor was there any evidence 
of an independent association between breast-feeding and verbal memory or visual search speed at 53 years.

It is suggested that the beneficial effect of breast-feeding on cognitive development results from the presence of certain essential long-chain fatty acids in breast milk, particularly arachidonic acid (AA) and docosahexaenoic acid (DHA) ${ }^{14}$. These favour the cerebral cortex and retina, accumulate during the last trimester from the placenta and during first year of life, and cannot be synthesised by infants.

We should emphasise that there was a disproportionate dropout over time of participants with low cognitive scores, which limits the generalisability of the study. We should also note that even though the effect of breastfeeding on cognition in midlife was statistically significant, the size of the effect was small (approximately a one-word difference on the NART between those never breast-fed and those breast-fed for 7 months or more), and its practical implications are unclear. On the other hand, the estimated 5\% difference in obtaining advanced educational qualifications between those of disadvantaged social background who were never breast-fed and those of similar background who were breast-fed for 7 months or longer has obvious functional significance, given the effect of advanced educational attainment on lifetime opportunity $^{3}$ and its potential for protection against serious cognitive decline ${ }^{15}$.

It is worth commenting on the apparent specificity of the breast-feeding effect on midlife cognition. Breastfeeding was independently associated with reading ability (NART) at age 53 years, but not with verbal memory or visual search speed. The NART employs 'irregular' words that can only be read correctly if the participant knows and recognises them in written form rather than relies on intelligent guesswork ${ }^{6}$. It thus represents a cognitive skill that depends on acquisition and use of information, sometimes referred to as crystallised intelligence ${ }^{16}$, as opposed to fluid intelligence, which enables the rapid solution of novel, unpractised tasks. The former is thought to be relatively stable across the adult life course and the latter more vulnerable to age-associated decline, a hypothesis for which there is support ${ }^{8}$. There was little evidence from the present study that breast-feeding differentially favoured the acquisition of crystallised intelligence in childhood, since at age 15 breast-feeding was positively associated with fluid-type skills (mathematics and AH4 non-verbal ability) as well as with verbal comprehension. The apparent sensitivity of adult wordreading performance to the effects of breast-feeding may therefore be due to the particular stability of this measure over time. Test scores for memory, speed and concentration, on the other hand, are likely to be more variable in midlife, subject to differing rates of age-related change, as well as to the influence of potentially labile factors such as mood and health. If so, we would not anticipate that breast-feeding would confer protection against clinically significant decline in memory and related cognitive skills, over and above its beneficial effect on education. The potential influence of breast-feeding on cognitive maintenance and decline in later life is, however, a matter for continuing investigation.

\section{Acknowledgements}

Funding for the NSHD is provided by the Medical Research Council. Data collection at 53 years was carried out by the National Centre for Social Research.

\section{References}

1 Anderson JA, Johnstone BM, Remley DT. Breast-feeding and cognitive development: a meta-analysis. Am. J. Clin. Nutr. 1999; 70: 525-35.

2 Rodgers B. Feeding in infancy and later ability and attainment: a longitudinal study. Develop. Med. Child Neurol. 1978; 20: 421-6.

3 Wadsworth MEJ. The Imprint of Time: Childhood, History and Adult Life. Oxford: Clarendon Press, 1991.

4 Wadsworth MEJ, Mann SL, Rodgers B, Kuh DL, Hilder WS, Yusuf EJ. Loss and representativeness in a 43 year follow-up of a national birth cohort. J. Epidemiol. Community Health 1992; 46: 300-4.

5 Department of Education and Science. Burnham Further Education Committee Grading Courses. London: HMSO, 1972.

6 Nelson HE, Willison JR. National Adult Reading Test (NART), 2nd ed. Windsor: NFER-Nelson, 1991.

7 Crawford JR, Stewart LE, Parker DM, Besson JAO, De Lacey G. Prediction of WAIS IQ with the National Adult Reading Test: cross-validation and extension. Br.J. Psychol. 1989; 28: 267-73.

8 Rabbitt P. Does it all go together when it goes? The Nineteenth Bartlett Memorial Lecture. Quart.J. Exp. Psychol. 1993; 46A: 385-434.

9 Masur DM, Sliwinski M, Lipton RB, Blau AD, Crystal HA. Neuropsychological prediction of dementia and the absence of dementia in healthy elderly persons. Neurology 1994; 44: 1427-32.

10 Jacobs DM, Sano M, Dooneief G, Marder K, Bell KL, Stern Y. Neuropsychological detection and characterization of preclinical Alzheimer's disease. Neurology 1995; 45: 957-62.

11 Pigeon DA. Details of the fifteen years tests. In: Douglas JWB, Ross JM, Simpson HR, eds. All Our Future. London: Davies, 1968; Appendix 1.

12 Office of Population Censuses and Surveys. Classification of Occupations. London: HMSO, 1970.

13 Douglas JWB. The Home and The School. London: Macgibbon \& Kee, 1964.

14 Crawford MA. The role of essential fatty acids in neural development: implications for perinatal nutrition. $\mathrm{Am}$. J. Clin. Nutr. 1993; 75(Suppl.): 703S-10S.

15 Stern Y, Gurland B, Tatemichi TK, Tang MX, Wilder D, Mayeux R. Influence of education and occupation on the incidence of Alzheimer's disease. J. Am. Med. Assoc. 1994; 271: 1004-10.

16 Horn JL, Cattell RB. Age differences in fluid and crystallized intelligence. Acta Psychologica 1967; 26: 107-29. 